\title{
Develop a GIS Based Mobile Application for Environmental Quality Survey and GPS for Weather Forecasting
}

\author{
Balasaheb R. Patil, Mayuri A. Patil \\ ${ }^{1}$ Environment science and engineering, KBC north Maharashtra University, Jalgaon. \\ ${ }^{2}$ Environment science and engineering, KBC north Maharashtra University, Jalgaon.
}

\begin{abstract}
Environmental Quality Surveys are used to measure the 'look and feel' of a location. The technique is very subjective, which means people will have different views about an area. Some will find a location unattractive, while others may find the same place very attractive.

An environmental quality survey uses an observer's judgements to assess environmental quality against a range of indicators. Sampling for environmental quality surveys within a study: Either an overall judgment of an area - walk around the whole Output Area and then complete the survey as a summary.

Weather forecasting is the application of science and technology to predict the conditions of the atmosphere for a given location and time. People have attempted to predict the weather informally for millennia and formally since the 19th century. Weather forecasts are made by collecting quantitative data about the current state of the atmosphere at a given place and using meteorology to project how the atmosphere will change.
\end{abstract}

Key Words: Microsoft Power BI, GPS mobile device, Google Maps and Power BI Analytics

\section{INTRODUCTION}

Environmental Quality Surveys are used to measure the 'look and feel' of a location. The technique is very subjective, which means people will have different views about an area. Some will find a location unattractive, while others may find the same place very attractive.

An environmental quality survey uses an observer's judgements to assess environmental quality against a range of indicators. Sampling for environmental quality surveys within a study: Either an overall judgment of an area - walk around the whole Output Area and then complete the survey as a summary.

Weather forecasting is the application of science and technology to predict the conditions of the atmosphere for a given location and time. People have attempted to predict the weather informally for millennia and formally since the 19th century. Weather forecasts are made by collecting quantitative data about the current state of the atmosphere at a given place and using meteorology to project how the atmosphere will change.
Environmental quality includes the natural environment as well as the built environment, such as air, water purity or pollution, noise and the potential effects which such characteristics may have on physical and mental health.

1) Air Quality

2) Water purity or pollution

3) Noise pollution quality

4) Weather quality aspects

It must be acknowledged that specifications (Software Requirement Specifications) are even more important if you're going to outsource software development. This is your guiding document, sacred tablets, and an ultimate protection insignia if any misunderstandings with the development team occur.

Methods for developing the mobile app or any software

a) Software development life cycle

1) Requirement gathering

2) Analysis

3) Design and algorithm

4) Coding or development

5) Testing

6) Deployment

\section{REVIEW OF LITERATURE}

\section{Environmental Quality Survey in Local Area}

I am investigating environmental quality in your local area. To do this I need a google account. Go through the process of setting up an account. To find out how to properly conduct an environmental quality survey in your local area To Explore the advantages and limitations and consideration of using environmental quality surveys Analysis on a particular area the find out the environmental measures through google map by mapping or plotting the layer side by side and second aspect also visit that place and make the analysis and compare the result as well or verify result on actual visit and google maps

\section{The Challenge}

Our challenge is to complete environmental quality surveys in selected local areas and present the findings on Google Maps. I can either visit the locations and complete the environmental quality surveys if it is safe to do so. However, this guide will take you through the steps to complete the environmental quality surveys using Google Street. There are limitations to this such as the images may have been taken some time ago, 
however, it will be good practice for completing environmental quality surveys.

\section{Why do an environmental Quality Survey}

Environmental Quality Surveys are used to measure the 'look and feel' of a location. It is a subjective method - one person may think a location is very noisy but another person may think that the same location is OK in terms of noise levels.

To identify the problems and damage in an urban area (e.g. quality of buildings, amount of traffic, lack of services and so on).

To Provide background information to establish how best to solve any problems and improve the surroundings of areas. To compare the environmental quality of two areas such as different zones of the city (CBD, inner city, suburbs), different villages, different tourist resorts or different shopping areas.

\section{Methodology and How its Work}

An environmental quality survey requires the use of a recording sheet with each category of the environment given a score between 1 and 5. For the reference as shown below figure.

Fig 1 Recording sheet

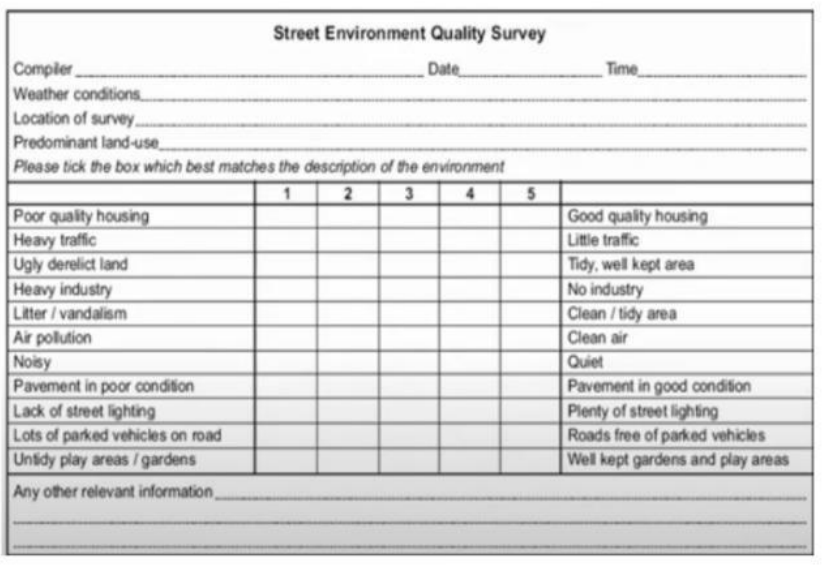

Fig 1 Recording sheet

Points are given for each feature by putting a tick in the appropriate column.

The points are then added up to give a total score for the environment.

The higher the total, the better the quality of the environment. $19 \mid \mathrm{P}$ a g e

At each of the locations selected for EQS, the environment of the area is scored for all the chosen categories.

For group work, judgment of quality should be calibrated so that all participants are scoring using the same standards.

The location of each survey should be carefully recorded on a base map or by noting the address.

The total score for all indicators should then be calculated at each survey site.

This process is repeated at the other selected location to allow comparisons of environmental quality to be made between different areas.
Photographs and detailed notes of any observations should be taken in your research area to analyze alongside your EQS results.

Field sketches can also be made to assist interpretations.

\section{Result Analysis}

\section{Bi-polar analysis}

Bi-polar analysis is a technique for comparing characteristics in different areas. This example shows how it can be used visually, using environmental quality survey results.

Pupils carried out an EQS of two different areas to allow for a comparison.

They rated the areas on a range of categories and on a scale of 1-5, with 5 being the highest score.

A dot showing this value is then placed on the diagram. Once this has been done for every category the dots are joined together and a visual comparison is then made by looking at lines as shown below diagram.
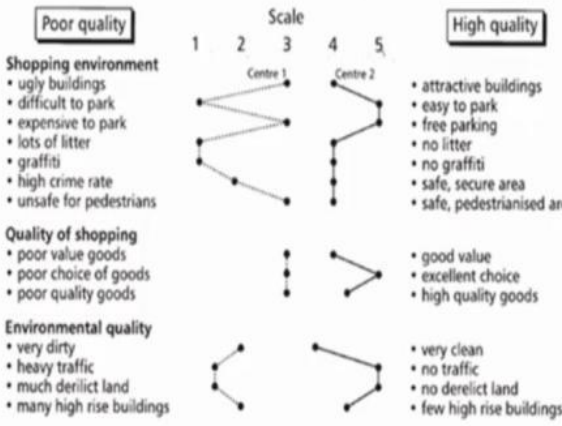
- ate, pedestrianised area

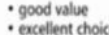
- high quality goods

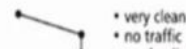
: no teaffe : no dereict land

Fig 2 Bi-Polar Analysis

\section{Weather and Weather Forecasting by GPS}

Weather forecasting is the application of science and technology to predict the conditions of the atmosphere for a given location and time. People have attempted to predict the weather informally for millennia and formally since the 19th century. Weather forecasts are made by collecting quantitative data about the current state of the atmosphere at a given place and using meteorology to project how the atmosphere will change.

Once calculated by hand based mainly upon changes in barometric pressure, current weather conditions, and sky condition or cloud cover, weather forecasting now relies on computer-based models that take many atmospheric factors into account.[1] Human input is still required to pick the best possible forecast model to base the forecast upon, which involves pattern recognition

skills, teleconnections, knowledge of model performance, and knowledge of model biases. The inaccuracy of forecasting is due to the chaotic nature of the atmosphere, the massive computational power required to solve the equations that describe the atmosphere, the error involved in measuring the initial conditions, and an incomplete understanding of atmospheric processes. Hence, forecasts become less accurate as the difference between current time and the time for which 
the forecast is being made (the range of the forecast) increases. The use of ensembles and model consensus help narrow the error and pick the most likely outcome.

There are a vast variety of end uses to weather forecasts. Weather warnings are important forecasts because they are used to protect life and property. Forecasts based on temperature and precipitation are important to agriculture, and therefore to traders within commodity markets. Temperature forecasts are used by utility companies to estimate demand over coming days. On an everyday basis, many use weather forecasts to determine what to wear on a given day. Since outdoor activities are severely curtailed by heavy rain, snow and wind chill, forecasts can be used to plan activities around these events, and to plan ahead and survive them.

Weather forecasting is a part of the economy, for example, in 2009 , the US spent approximately $\$ 5.1$ billion on weather forecasting, producing benefits estimated at six times as much. To find the weather of a particular place by using weather API and geolocation plugins in mobile device with help of GPS With the help of GPS device, we know the current location of a particular place and we get the geo spatial data in the form of latitude and longitude

By providing latitude and longitude we can get the accurate weather condition of that place by using weather API

\section{DESIGN AND DEVELOPMENT}

\section{Requirement Analysis and Technology}

Requirement Analysis, also known as Requirement Engineering, is the process of defining user expectations for a new software being built or modified. In software engineering, it is sometimes referred to loosely by names such as requirements gathering or requirements capturing. Requirement's analysis encompasses those tasks that go into determining the needs or conditions to meet for a new or altered product or project, taking account of the possibly conflicting requirements of the various stakeholders, analyzing, documenting, validating and managing software or system requirements. Here are the objectives for performing requirement analysis in the early stage of a software project

\section{Software Requirements}

Software requirements break-down the steps needed to meet the business requirement or requirements. Whereas a business requirement states the 'why' for a project, a software requirement outlines the 'what'. For example, if the business requirement is to create a member directory for a trade association, the software requirements will

27 | $\mathrm{P}$ a g e

outline who has access to the directory, how members register with the directory, who will have ownership of the data, what vehicle or vehicle will be used such as a website or paperbased directory, and so on.

\section{Data Flow Diagram}

A Data Flow Diagram (DFD) can be designed early in the requirement elicitation process of the analysis phase within the SDLC (System Development Life Cycle) to define the project scope. A DFD is often used as a preliminary step to create an overview of the system without going into great detail, which can later be elaborated.

For instance, if there is a need to show more detail within a particular process, the process is decomposed into a number of smaller processes in a lower level DFD. In this way, the Content Diagram or Context-Level DFD is labeled a "Level-0 DFD" while the next level of decomposition is labeled a "Level-1 DFD", the next is labeled a "Level2 DFD", and so on.

Requirement of the resources

1) Open Layer API.

2) Google Map Plugin.

3) Geolocation Plugin.

4) Voice Recognition Plugin.

5) Analysis on Microsoft Power BI tools (Integrate in Ionic with coding).

6) Learn about map analysis with the help of my google map.

7) Weather API for weather forecasting.

8) Gather information from the Internet.

Technology Specification

1) Hardware requirement Laptop for coding or computer with 4GB RAM and 250 hard disks Operating system - Windows 10 Android Mobile - for application deployment and testing.

2) Software Requirement - Visual Studio Code editor Node JS command Prompt Ionic Framework Java framework SQL server Android Studio SDK .

3) Technology Front End - Ionic Framework and Angular for UI (User Interface) Backend - MS SQL server for storage purpose and C\#.net for API development .

\section{Design and Architecture Diagram}

I followed the agile fundamentals principles to develop the software.

Generally, I developed application in two parts and actually three parts

1) Environmental Quality Survey (EQS)

2) Weather Forecasting by GPS and Weather API

3) Microsoft Power BI Reports

In the 1st part we are getting the current geo spatial coordinates by default or we have already provided the get current location button on the header bar of the application.

The current location is in the form of latitude and longitude. Then we put up the one navigation button for My google map, after clicking on that button google map is open and there we can do the analysis on a particular site or location.

There is the provision for saving the data in the form of layers and displaying it and which is managed by the coding.

Now here is block diagram for part $1 \mathrm{EQS}$ as shown below 


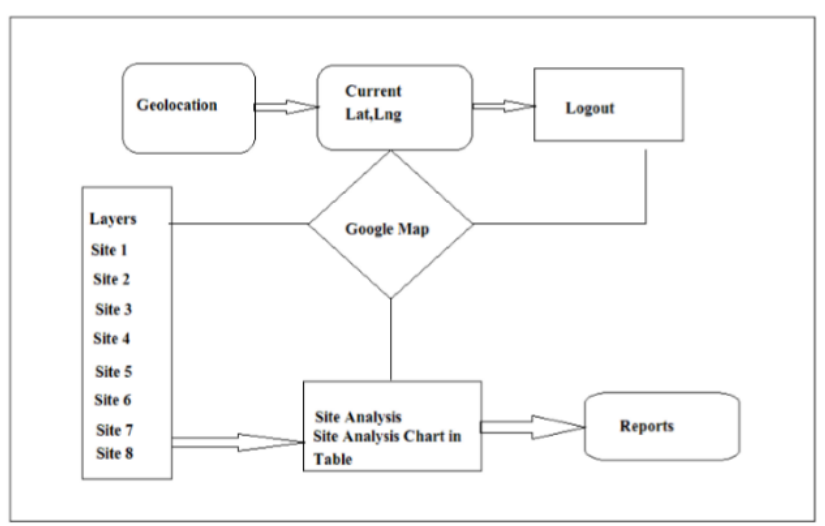

Fig EQS Architecture

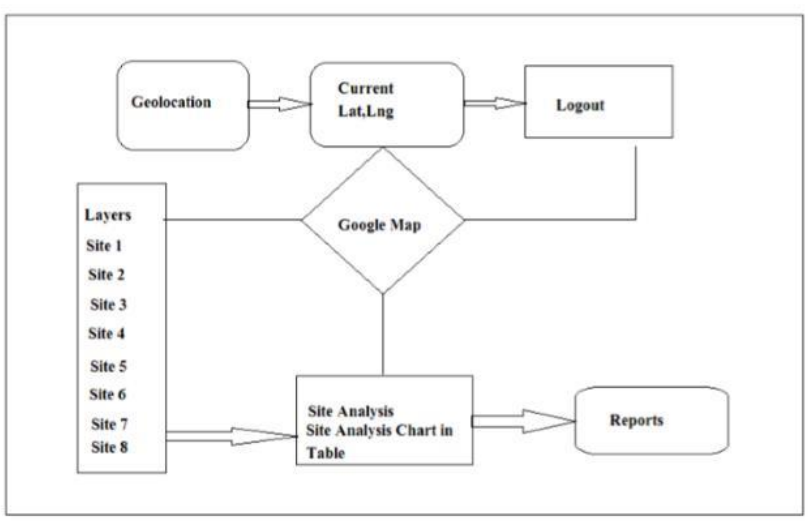

Fig EQS Architecture

From the diagram I am doing the analysis on the different locations and recording the result in the form number in the record sheet.

From the record sheet I save those data in the database and I create the report on the basis of those data in Microsoft Power BI tool for advanced AI based reporting.

I also have the second option to store the data in excel sheet and create the report in Power BI by using excel sheet.

I am able to display the report in the form of an advanced AI based chart system. This is the main feature of the application in the reporting.

Now Let's discuss on Use case diagram of the EQS as shown below

Now move on to the second part of the Application that is Weather forecasting by using geolocation and Open Layer weather API and Managed the data and smart searching.

The challenging part of this section is to integrate the Voice recognition algorithm for advanced AI based weather forecasting.

Here I am creating the three ways for weather forecasts; one is getting the weather by default, current location on current date time and next 10 days weather forecast.

Second is search by the city name enter the city name in the textbox then result is display on the basis on current date and time and forecasting for next 10 days weather condition.

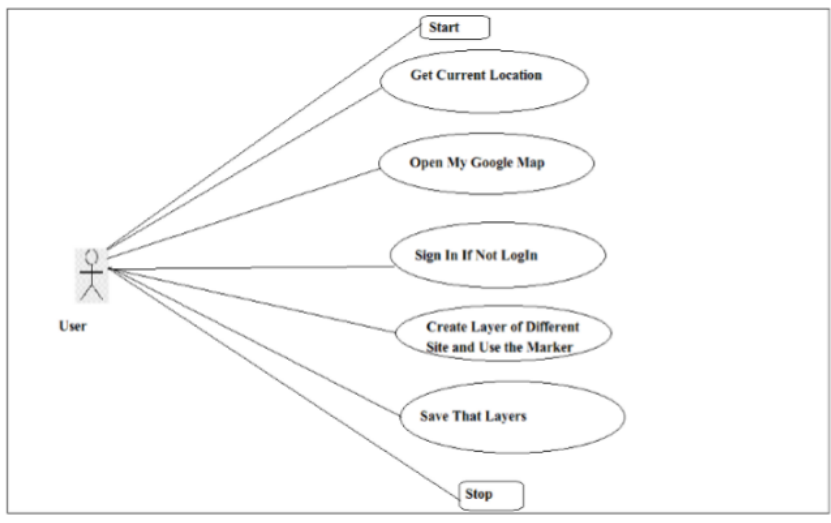

Fig Use case diagram for EQS

Third part is the get weather of the particular location by Voice recognition technique the method is the simply speak the quote 'A weather for Jalgaon City' as per voice recognition this above text is display and with help of this text, we can search the result by using AI algorithm and get the weather condition of current location and date time and next 10 days forecast.

The architecture diagram for weather forecasting as shown below diagram.

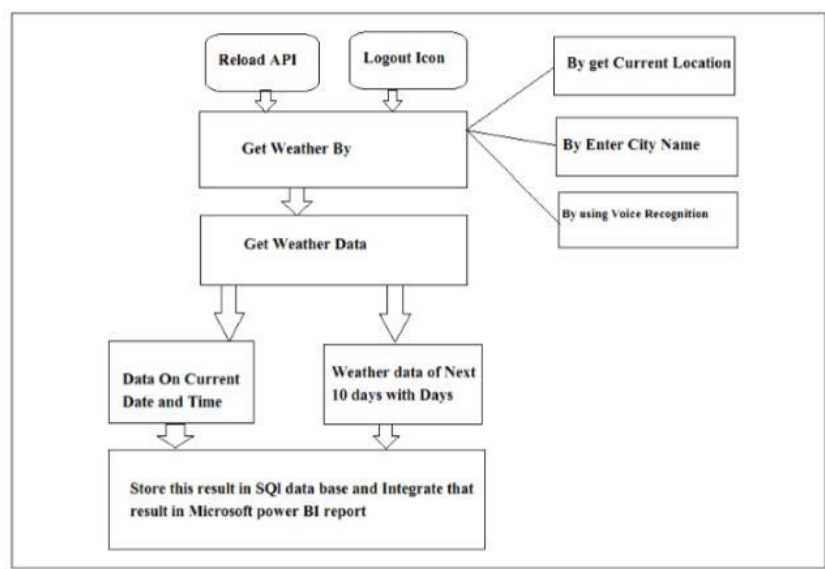

Fig Architecture Diagram for Weather Forecast

From the architecture diagram we clearly understand the working of part 2 , how the get the weather result, the next diagram for internal API flow for the Weather forecasting .

\section{DEVELOPMENT AND CODING}

This is the very important step of the development phase after designing a particular module by following agility principles.

I followed the singleton design pattern.

Singleton is a creational design pattern, which ensures that only one object of its kind exists and provides a single point of access to it for any other code. Singleton has almost the same pros and cons as global variables. Although they're super-handy, they break the modularity of your code. 
Part 1 - Environmental Quality Survey Module Coding UI Module

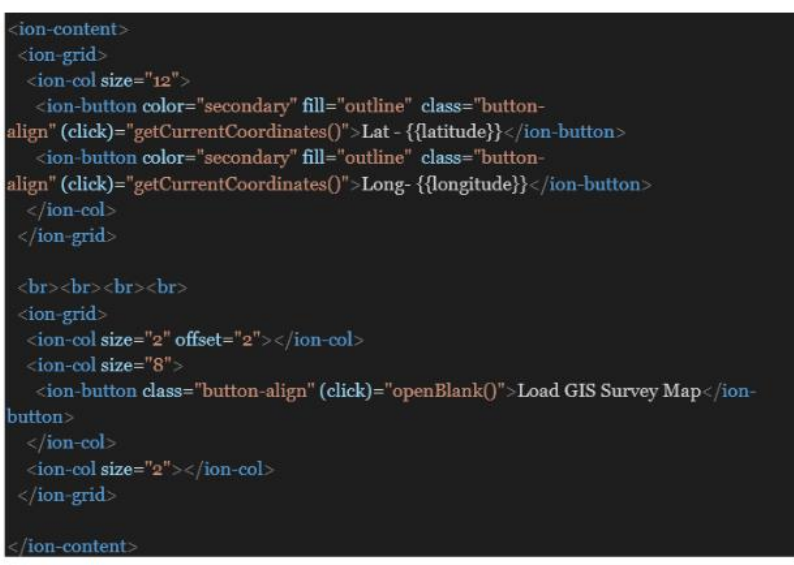

Part 2 - Weather Forecasting

UI-Module

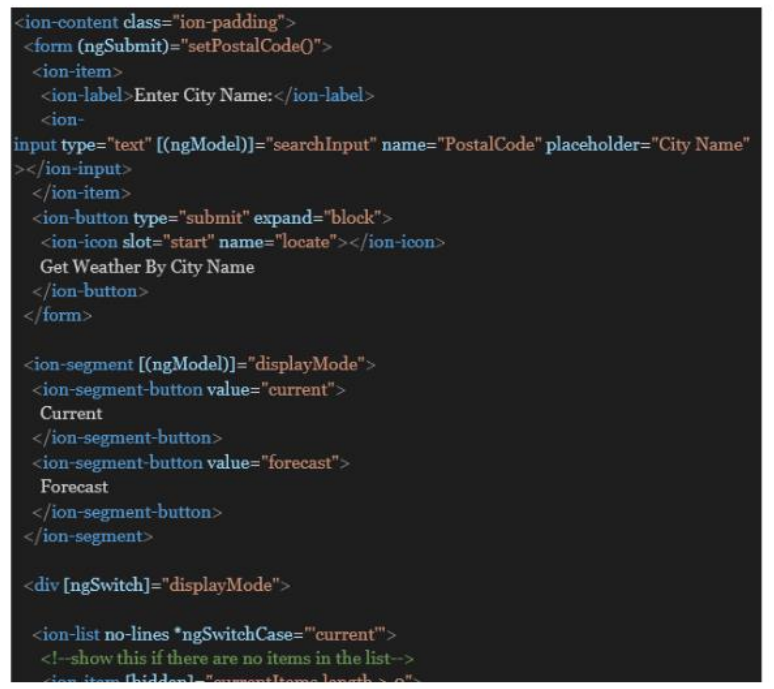

\section{Testimonials and Practical's}

\section{A) EQS By My Google Map}

Testimonials sample analysis for seven locations.

While EQS sections load it gets latitude and longitude coordinates by GPS.

While clicking on the Load GIS survey map button then Map is open, In map I create the layers for quality survey and analysis on seven locations as shown below figures.

As per above diagram, here are three options.

1.Add Layer

2.Share

3.Preview

After clicking on the preview button then we can see the analysis layer of quality survey.
Testimonials sample analysis for seven locations.

EQS By My Google Map
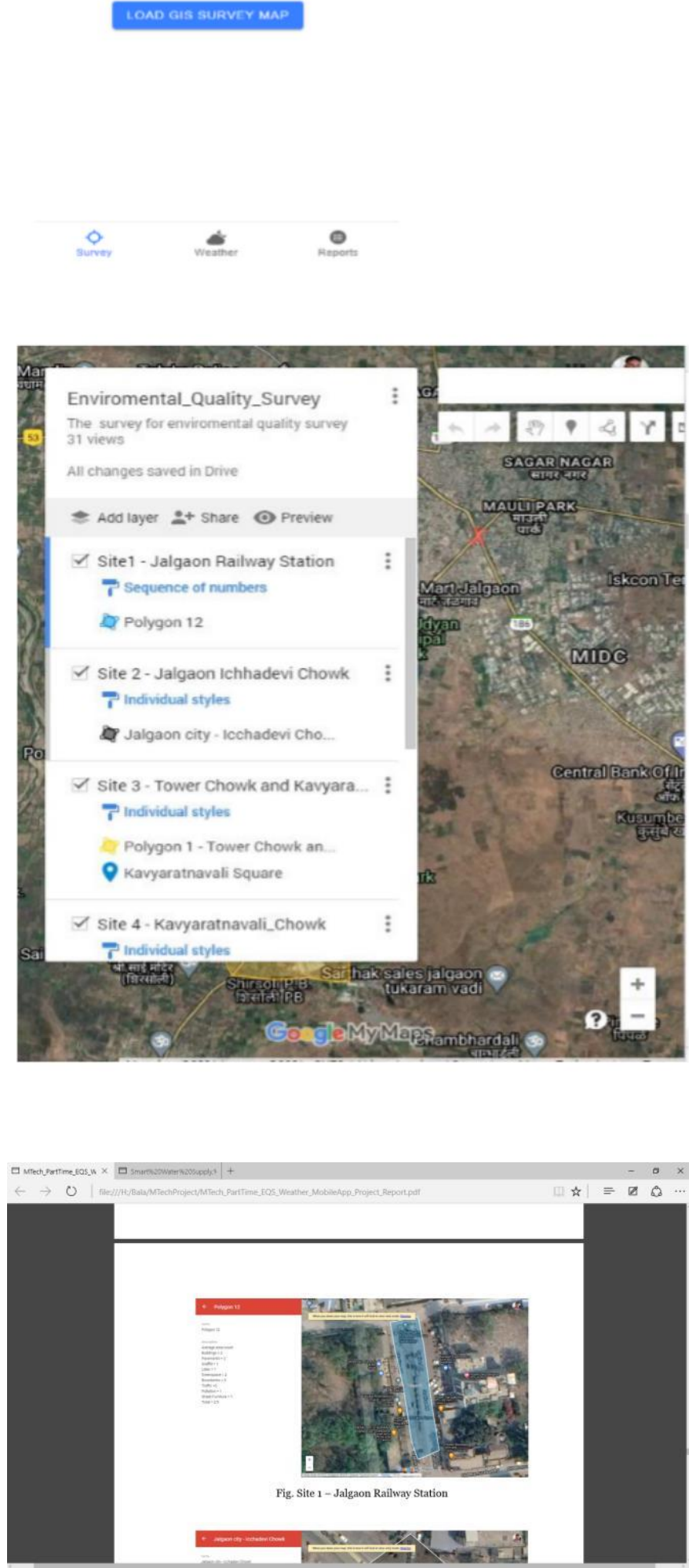


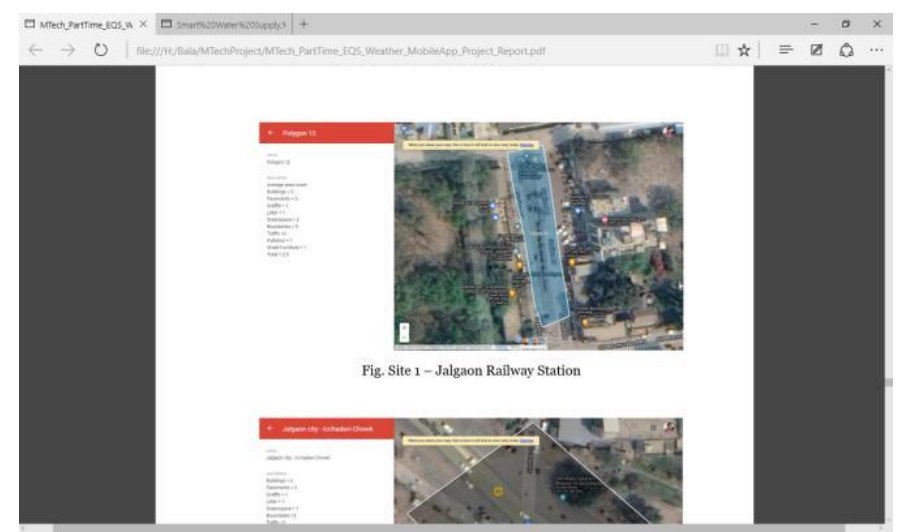

B) Weather Forecasting Section

Weather forecasting by three ways

1.Get Weather by Current Location.

2.Get Weather by City Name

3.Get Weather by Voice Recognition

This is about getting weather on current date and time now next section is forecasting on current search weather.

The weather forecasting for hourly on current date for 1 days or next 10 days

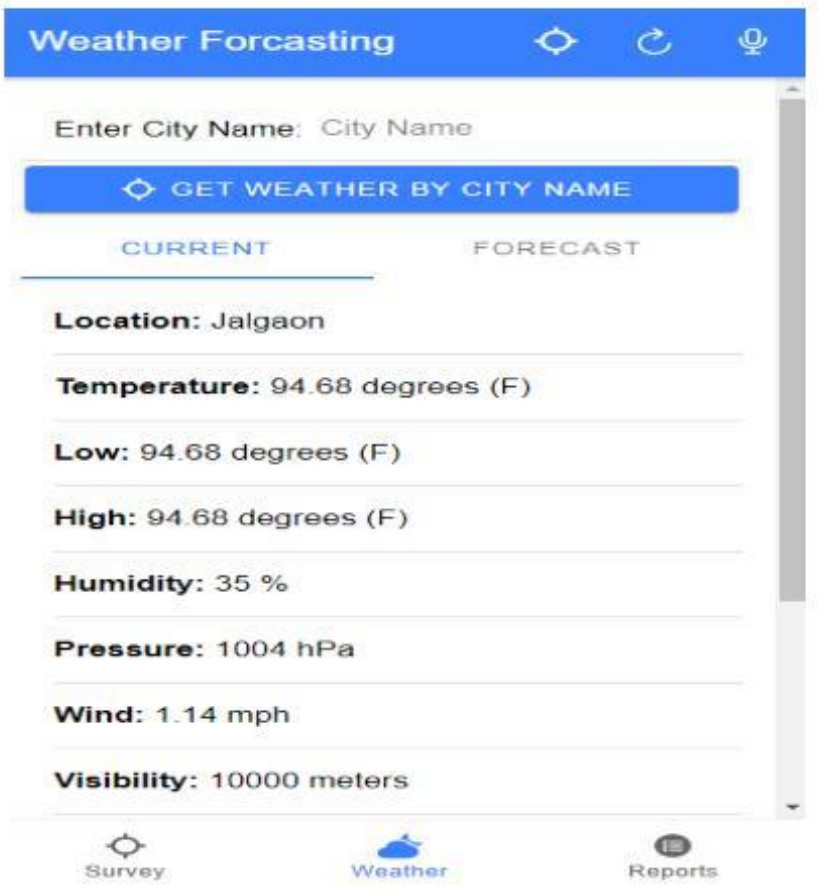

\section{Fig. Get Current Tab Weather}

\section{Deployment}

Deployment is the last step of software development and this is performed on client machine or mobile after testing phase Here developers generate the APK file for android device and the end user is installed directly on the phone or it will be published on google play store.

For App upload on google play store end user must have to google play store account.

\section{RESULTS AND DISCUSSION}

In this section we are give the result as per our analysis regarding EQS

Here is the two view of result display

1)Record Result on Manual Sheet.

2)Display Result by Microsoft Power BI tools in mobile as well as desktop view.

Now we proceed with manual sheet

Site1 - Jalgaon Railway Station

Table -1: Sample Table format

\begin{tabular}{|c|l|r|r|r|r|r|}
\hline \multicolumn{6}{|c|}{ Site Name - Site1 - Jalgaon Railway Station (Area 174 meter) } \\
\hline No & \multicolumn{1}{|c|}{ Parameter } & 1 & 2 & 3 & 4 & 5 \\
\hline 1 & Buildings & & 2 & & & \\
\hline 2 & Pavements & 1 & & & & \\
\hline 3 & Garffiti & 1 & & & & \\
\hline 4 & Litter & & & & 4 & \\
\hline 5 & Greenspaces & & 2 & & & \\
\hline 6 & Boundaries & & & 3 & & \\
\hline 7 & Traffic & & 2 & & & \\
\hline 8 & Pollution & & & 3 & & \\
\hline 9 & Street Furniture & & 2 & 3 & & \\
\hline $\begin{array}{l}\text { Remark - This area has large pollution, traffic and less } \\
\text { greenspaces }\end{array}$
\end{tabular}

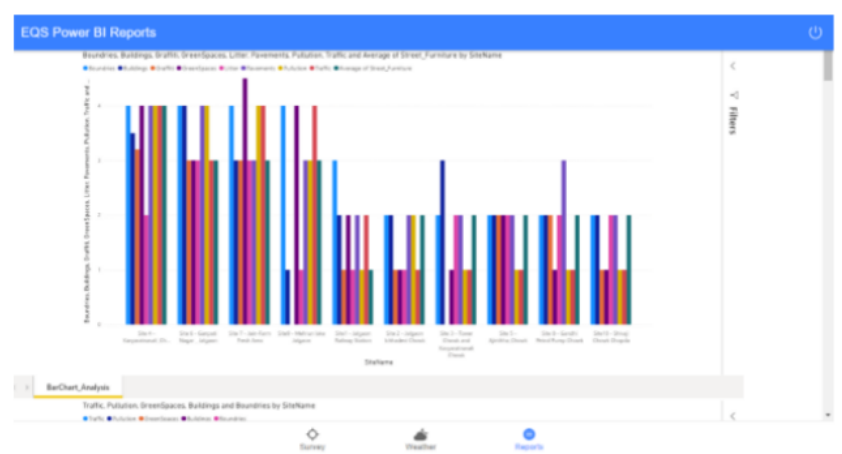

Fig. Bar-Chart Analysis for all sites

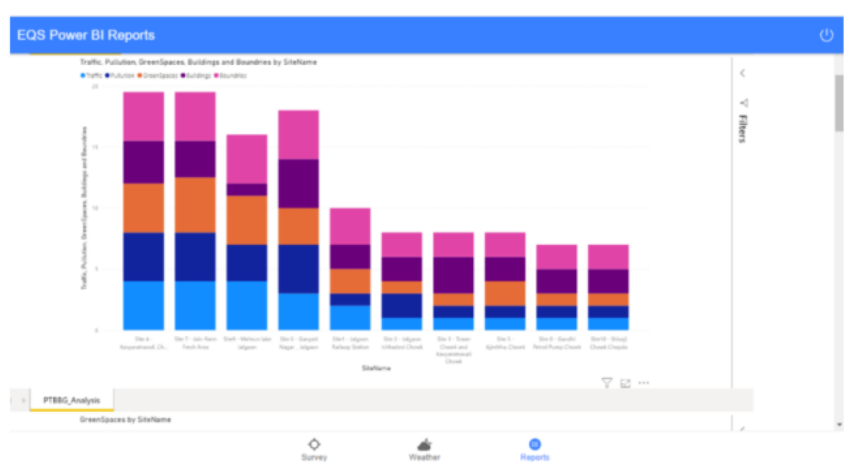

Fig. PTBG Analysis 


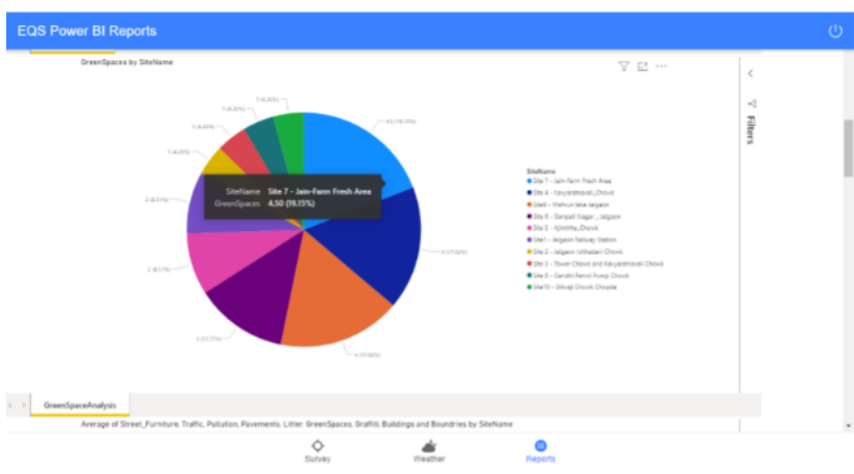

Fig. Green spaces analysis for all Sites

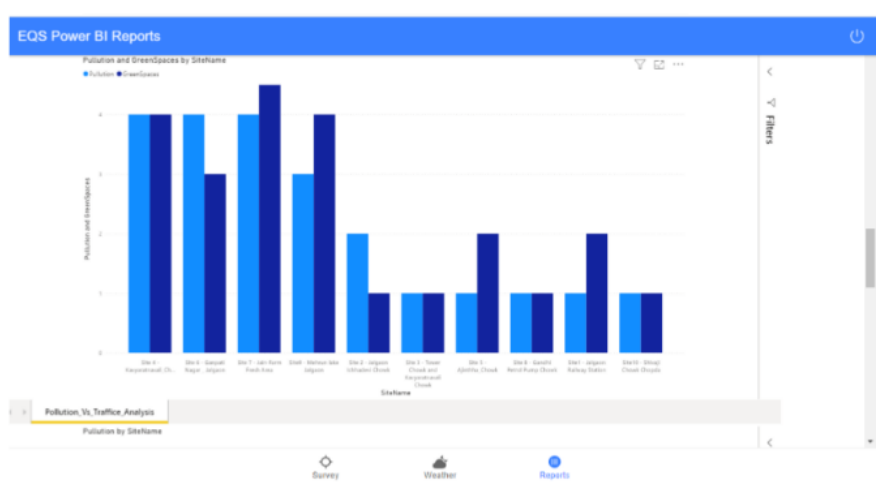

Fig. pollution Vs Traffic Analysis

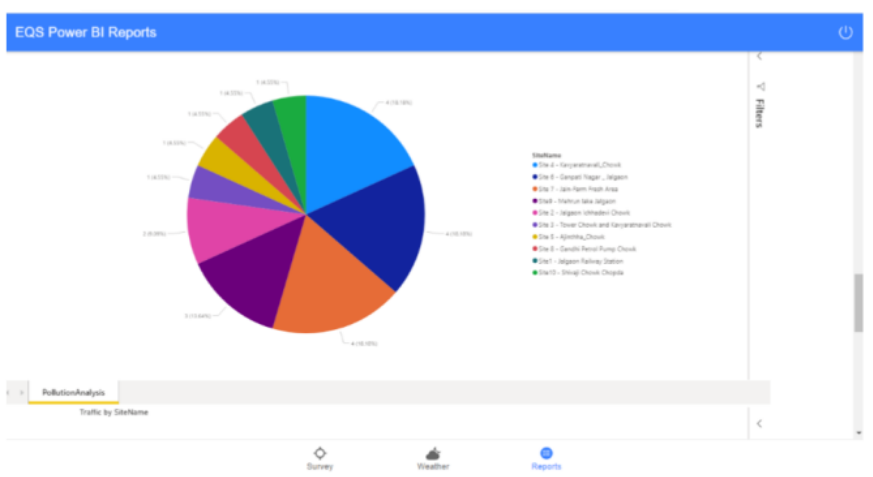

Fig. pollution analysis

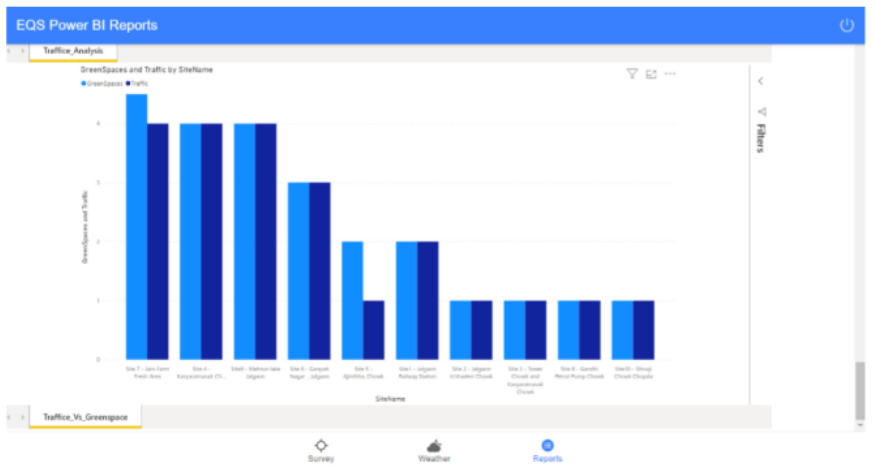

Fig. Traffic Vs Green space analysis

\section{CONCLUSION}

This is to conclude analysis on EQS and Weather forecasting data management by using advanced reporting tools and AI (Artificial Intelligence for data automation) based Power BI
(Microsoft Business Intelligence tool for advanced reporting) report Integration with web as well as mobile application for displaying the result.

Nowadays this is not a time to make analysis on environmental fields \& saved data on excel or any other resources, which is very time consuming to explore the result to the end user but using automation and data science techniques we can achieve it in a smart way.

Study of environmental quality survey and weather forecasting and integration with digital platforms by using advanced techniques that are Microsoft Power BI and Voice recognition in weather forecasting.

\section{FUTURE SCOPES}

Nowadays each and everything is going digital and change is the need of hour.

The future scope regarding EQS we can implement the tracking devices for pollution or air quality and make improvements in reporting and live data management by using artificial intelligence.

Nowadays it already exists, a tracking device for checking the quality of the environment but issues in reporting and live data management. It is work when we measure or we need but our vision is to test it automatically as per quality priority and give the alert for future events.

\section{REFERENCES}

1)Get the reference and gather information from Elsevier journal Global Ecology and Conservation Journal - Elsevier.

2) Get the information from internet geography site https://www.internetgeography.net/google-my-mapsproj ectenvironmental-quality-survey-in-my-local-area/.

3) Requirement analysis from the Internet.

4) Integrate the advanced level reporting by using Microsoft power BI.

5) Get Ionic Cordova references for Voice recognition plugins and Geolocation plugins.

6) Study of Microsoft Power BI tool and integrate that services in our application by coding support on https://docs.microsoft.com/enus/rest/api/power-bi/

7) Learn the report customization techniques on https://docs.microsoft.com/en-us/power-bi/

8) Study and Integrate the GPS plugins in application by using following documentation -

https://ionicframework.com/docs/native/geolocation

9) Study of google map API https://console.cloud.google.com/google/maps-apis/over view and https://www.google.com/maps/d/u/0/ .

10) project source code and backup is uploaded on git account.

https://github.com/BalaPatil92/GISGPS_Weather 